\title{
Independent Predictors of Severity and Functional Outcome of Acute Ischemic Stroke in Patients with Diabetes
}

\author{
Sai Sampath Kumar Natuva ${ }^{{ }^{*}}$, Sunanda Tirupati ${ }^{2}$, Amaresh P. Reddy ${ }^{2}$, Ganesh Vallampalli ${ }^{1}$ and Sangamithra Gandra ${ }^{1}$ \\ ${ }^{1}$ Department of Neurology, Narayana Medical College, Nellore, Andhra Pradesh, India \\ ${ }^{2}$ Department of Endocrinology, Narayana Medical College, Nellore, Andhra Pradesh, India
}

Correspondence to:

Sai Sampath Kumar Natuva, DM

Department of Neurology

Narayana Medical College, Nellore-524003

Andhra Pradesh, India

E-mail: drnssampath123@gmail.com

Received: May 06, 2016

Accepted: July 08, 2016

Published: July 12, 2016

Citation: Natuva SSN, Tirupati S, Reddy AP, Vallampalli G, Gandra S. 2016. Independent Predictors of Severity and Functional Outcome of Acute Ischemic Stroke in Patients with Diabetes. J Neurol Exp Neurosci 2(1): 15-20.

Copyright: () 2016 Natuva et al. This is an Open Access article distributed under the terms of the Creative Commons Attribution 4.0 International License (CC-BY) (http://creativecommons. org/licenses/by/4.0/) which permits commercial use, including reproduction, adaptation, and distribution of the article provided the original author and source are credited.

Published by United Scientific Group

\begin{abstract}
Background: Diabetes mellitus the metabolic disorder, can interact with atherosclerosis in ischemic strokes to initiate, activate and propagate vascular events Patients with diabetes have a two- to threefold higher risk of developing atherosclerotic vascular disease. This increased risk is largely attributed to the clustering of other risk factors like, age, hypertension, dyslipidemia, poor glycaemic status.
\end{abstract}

Purpose of the Study: To identify the independent predictors for severity and functional outcome of acute ischemic stroke in patients with diabetes mellitus.

Materials and Methods: The study is a prospective, case-control, hospital based study done at Department of Neurology, Narayana Medical College for a period of $1 \frac{1}{2}$ year.

Results: 100 acute ischemic stroke patients were recruited in the study and the distribution of risk factors among them were studied. Age, smoking, alcohol, CV Doppler, TGL, HDL, FPG, PPG and HbA1c were found to have cumulative influence on the stroke severity (NIHSS) and functional outcome (mRS). Whereas alcohol and HbA1C found to have significant independent predictive value for stroke severity at admission.

Conclusion: Alcohol and $\mathrm{HbA1c}$ at admission were found to have independent effect on the stroke severity. None of the risk factors studied were having independent impact on the stroke functional outcome at the follow up.

\section{Keywords}

Risk factors, Severity, Outcome, Ischemic stroke, Diabetes, HbA1C

\section{Introduction}

Stroke is a global health problem. Currently, ischemic heart disease and stroke are the leading causes of mortality worldwide and more than $80 \%$ of deaths occurring in the low and the middle income countries [1].

The risk factors for stroke are modifiable and non-modifiable. The modifiable risk factors are mostly related to the atherosclerotic burden and include diabetes, hypertension, smoking, and hyperlipidemia [2]. Stroke is responsible for 3 million deaths and is rising in developing countries [3], and is a major cause of mortality and morbidity in Asian countries.

In most of the ischemic strokes the underlying pathophysiology is atherosclerosis, which is a chronic inflammatory disorder in which immune mechanisms interact with modifiable metabolic risk factor like diabetes mellitus 
to initiate, activate and propagate vascular events. Diabetes mellitus is a very common metabolic disorder with high and increasing prevalence worldwide.

Risk factors for macrovascular disease in diabetic individuals include dyslipidemia, hypertension, obesity, reduced physical activity, and cigarette smoking. Additional risk factors more prevalent in the diabetic population include microalbuminuria, macroalbuminuria, an elevation of serum creatinine, and abnormal platelet function.

This increased risk of ischemic stroke is largely attributed to the clustering of individual risk factors rather than from the separate vascular risk factors.

The aim of the study is to identify the independent predictors for severity and functional outcome of acute ischemic stroke in patients with diabetes mellitus.

\section{Materials and Methods}

The present study was done at department of Neurology, Narayana medical college, Nellore for a period of $1 \frac{1}{2}$ year from January 2014 to June 2015. The current study was a prospective case-control, hospital based study and was approved by the institute ethics committee of Narayana medical college and hospital. The study recruited the patients of Endocrinology, Neurology OPDs and Emergency departments.

A total of 100 Acute ischemic stroke patients with diabetes were included. They were categorised into 2 sub groups based $\mathrm{HbA1c}$ (glycated haemoglobin) level at the time of admission as acute ischemic stroke patients with diabetes with good glycemic control (< 7 HbA1c) and acute ischemic stroke patients with diabetes with poor glycemic control $(>7$ HbA1c).

\section{Inclusion criteria}

Acute ischemic stroke patients

\section{Exclusion criteria}

Intra cerebral haemorrhage, Space occupying lesions, Sub arachnoid haemorrhage, Cerebral venous thrombosis, Transient ischemic attacks and Patients with recurrent cerebrovascular events.

A proforma was prepared which includes detailed history clinical examination and requisite investigations available at Narayana hospital. History includes all of the symptoms pertaining to the ischemic stroke in detail with emphasis on all the risk factors including the glycemic status at the time of admission.

Glycemic status in acute ischemic stroke patients with diabetes was evaluated by measuring the HbA1c level and fasting and post prandial plasma glucose levels. The stroke severity at admission was assessed based on NIHSS (National institute of health sciences scale) and patients were followed up to discharge and 3 months subsequently. NIHSS cut off value of 8 was taken to classify the severity of the stroke, a score of $>8$ was taken as moderate to severe stroke and $<8$ was taken as mild stroke. The stroke functional outcome at 3 months was assessed by mRS (modified Rankin score). mRS scores $0-2$ were considered as independent functional outcome and 3-6 were considered as dependent functional outcome.

\section{Statistical Analysis}

The data values have been entered into MS - Excel and statistical analysis has been done by using IBM SPSS Version 20.0. For categorical variables the values are represented as number and percentages. To test association between the groups, chi-square test has been used. For continuous variables, the values are represented as mean and standard deviation. To test the mean difference between three or more groups, ANOVA (Analysis of variance) test with post hoc (Tukey's) test has been used. All the $\mathrm{p}$ values are having less than 0.05 are considered as statistically significant. Multivariate analysis has been done to identify the independent predictors among the studied risk factors.

\section{Results}

Total number of patients analysed for the study were 100 acute ischemic stroke patients with diabetes. Males were predominant (72/100) comprising $72 \%$ of study population. Mean age of patients was $53.78 \pm 12.679$ years. Non smokers (58\%) were more when compared to smokers (42\%). Most of the patients were non alcoholics (69\%). Hypertension was noted in $68 \%$ of patients (Table 1 ).

Significant hemodynamic changes of carotid/vertebral doppler (> 50\% stenosis \& flow dynamics) were noted in 16 patients $(12.3 \%)$ out of 100 acute ischemic stroke patients. 97 patients had large artery to artery atherosclerotic etiology. Where as 3 patients had cardioembolic etiology. LVH (left ventricular hypertrophy) was observed in 33\% of patients. Good gycemic status ( $\mathrm{HbA} 1 \mathrm{C}<7)$ was observed in $60 \%$ of patients and poor glycemic status $(\mathrm{HbA} 1 \mathrm{C}>7)$ was observed in $40 \%$.

\section{Stroke severity at admission (NIHSS)}

There was significant correlation $(\mathrm{P}<0.05)$ for age, sex, smoking and alcoholism with stroke severity in ischemic stroke patients with diabetes. Hypertension did not have positive correlation among patients with severity of stroke. Significant hemodynamic abnormalities on carotid/vertebral doppler were not having any statistical association with the severity of stroke. TOAST classification for etiology has no significant statistical distribution. $60 \%$ of patients of poor glycemic control were smokers whereas nearly $70 \%$ of patients of good glycemic control were non smokers. Similarly, majority of alcoholic patients were from poor glycemic control (Table 1).

Total cholesterol is high in $42 \%$ of Patients and it is correlated with severity of stroke (P-0.017). VLDL (very low density lipoprotein) and TGL (triglycerides) also found to have statistical correlation with stroke severity ( $\mathrm{p}$ values $0.03 \& 0.001$ ). HDL (high density lipoprotein), LDL (low density lipoprotein) and TG/HDL ratio did not found to have statistically significant association. 
Table 1: Categorical and Continuous variables (univariate analysis) for predictors of stroke severity (NIHSS) at admission.

Categorical variables (univariate analysis) for predictors of stroke severity (NIHSS) at admission

\begin{tabular}{|c|c|c|c|c|c|c|}
\hline & \multirow{3}{*}{$\begin{array}{l}\text { was noted in patients with poor glycemic control. TSH values } \\
>5 \text { were observed in } 42 \text { patients and it had no significant } \\
\text { correlation with the severity of stroke. }\end{array}$} \\
\hline S.No & Variable & & $\begin{array}{c}\text { NIHSS } \\
<=8\end{array}$ & $\begin{array}{c}\text { NIHSS } \\
>8\end{array}$ & P-Value & \\
\hline & & $\mathrm{F}$ & 19 & 9 & & \\
\hline
\end{tabular}

Stroke functional outcome at 3 months (mRS)

Age, smoking, alcohol, carotid doppler, HDL in males, TGL, TGL/HDL ratio, FPG, PPG and HbA1c at admission were found to have statistically significant correlation with the stroke functional outcome (mRS) at 3 months (Table 2).

Older age patients were more prone for poor functional outcome with $\mathrm{mRS}$ values in between 3-6 (P-0.028). Smoking and alcoholism is associated with poor functional outcome with $p$ values $0.02 \& 0.029$ respectively. High FPG and high PPG values were also associated with poor stroke functional outcome with very high statistical significance, $p$ values being 0.002 for both the variables. Significant percentage of good glycemic control group (95\%) patients had functional independence at 3 months of stroke follow up. Proportionally $47.5 \%$ of poor glycemic control patients had functional dependence at 3 months of stroke follow up. HDL value in males had statistically correlated with functional outcome (P0.038). Among the dyslipidemias triglycerides found to have statistical association with the functional outcome (P-0.01). Though carotid doppler was not found to have statistical correlation with the severity of stroke, but it had significant statistical correlation with the functional outcome (P-0.003). Sex, hypertension, $\mathrm{LVH}$ and TSH values did not have positive correlation among diabetic patients. Other than TGL, HDL other dyslipidemic states were not found to have significant effect on functional outcome.

Multivariate analysis (Table 3) of all the risk factors was done to recognize the impact and statistical significance of individual factor for the stroke severity at admission and functional outcome of stroke at the follow up of 3 months. None of the factors found to have independent predictive value for the stroke functional outcome. Whereas alcohol (P$0.001)$ and $\mathrm{HbA} 1 \mathrm{c}$ at admission $(<0.0001)$ were found to have significant predictive value for stroke severity at admission.

\section{Discussion}

Ischemic stroke is a heterogeneous pathophysiological state in which varied different pathways might lead to indistinguishable clinical presentations that result in high mortality rates and severe disabilities. Well recognized etiologies of ischemic stroke include cardiac or artery-toartery embolism and atherothrombosis of an extracranial, intracranial carotid and vertebral artery systems due to Type 2 DM. However, it is generally accepted that atherosclerosis of extracranial or intracranial arteries due to Type $2 \mathrm{DM}$ accounts for a substantial proportion of clinical ischemic strokes.

This is one of few clinical studies where the strenuous effort had been made to identify the independent predictors 
Table 2: Categorical and continuous Variables (univariate analysis) for predictors of functional outcome of Ischemic stroke at 3 months.

Categorical Variables (univariate analysis) for predictors of functional outcome of Ischemic stroke at 3 months

\begin{tabular}{|c|c|c|c|c|c|}
\hline S.No & Variable & & $\begin{array}{c}\text { MRS } \\
\text { 0-2 }\end{array}$ & $\begin{array}{c}\text { MRS } \\
3-6\end{array}$ & P-Value \\
\hline \multirow{2}{*}{1} & \multirow{2}{*}{ Sex } & $\mathrm{F}$ & 23 & 5 & \multirow{2}{*}{0.533} \\
\hline & & M & 55 & 17 & \\
\hline \multirow{2}{*}{2} & \multirow{2}{*}{ Smoking } & NO & 50 & 8 & \multirow{2}{*}{${ }^{*} 0.02$} \\
\hline & & YES & 28 & 14 & \\
\hline \multirow{2}{*}{3} & \multirow{2}{*}{ Alcohol } & NO & 58 & 11 & \multirow{2}{*}{ *0.029 } \\
\hline & & YES & 20 & 11 & \\
\hline \multirow{2}{*}{4} & \multirow{2}{*}{ HTN } & $\mathrm{NO}$ & 24 & 8 & \multirow{2}{*}{0.619} \\
\hline & & YES & 54 & 14 & \\
\hline \multirow{2}{*}{5} & \multirow{2}{*}{ 2D Echo } & LVH & 22 & 10 & \multirow{2}{*}{0.32} \\
\hline & & NO LVH & 56 & 12 & \\
\hline \multirow{2}{*}{6} & \multirow{2}{*}{$\begin{array}{l}\text { Carotid } \\
\text { Doppler }\end{array}$} & NO SIG & 70 & 14 & \multirow{2}{*}{${ }^{*} 0.003$} \\
\hline & & SIG & 8 & 8 & \\
\hline \multirow{2}{*}{7} & \multirow{2}{*}{ Toast } & Cardioembolic & 2 & 1 & \multirow{2}{*}{0.63} \\
\hline & & LA & 76 & 21 & \\
\hline \multirow{2}{*}{8} & \multirow{2}{*}{$\begin{array}{c}\text { Total Cho- } \\
\text { lesterol }\end{array}$} & $<=200$ & 45 & 13 & \multirow{2}{*}{0.907} \\
\hline & & $>200$ & 33 & 9 & \\
\hline \multirow{2}{*}{9} & \multirow{2}{*}{ HDL(M) } & $<=50$ & 33 & 14 & \multirow{2}{*}{${ }^{*} 0.038$} \\
\hline & & $>50$ & 17 & 1 & \\
\hline \multirow{2}{*}{10} & HDI (F) & $<=40$ & 4 & 2 & 0264 \\
\hline & IIDL(F) & $>40$ & 19 & 3 & 0.204 \\
\hline & & $<=100$ & 30 & 9 & \\
\hline 11 & Hot & $>100$ & 48 & 13 & 0.035 \\
\hline & & $<=30$ & 44 & 8 & \\
\hline & VEDL & $>30$ & 34 & 14 & 0.096 \\
\hline 12 & TCI & $<=150$ & 49 & 7 & *001 \\
\hline 13 & $1 G L$ & $>150$ & 29 & 15 & 0.01 \\
\hline 14 & TCI/UDI & $<=3$ & 42 & 8 & 140 \\
\hline 14 & I UL/TH & $>3$ & 36 & 14 & 0.140 \\
\hline 15 & FBS & $<=130$ & 59 & 9 & $* 0002$ \\
\hline & & $>130$ & 19 & 13 & 0.002 \\
\hline 16 & DPPC & $<=180$ & 59 & 9 & $* 0000$ \\
\hline 10 & 1003 & $>180$ & 19 & 13 & 0.002 \\
\hline 17 & TSH & $<=5$ & 48 & 10 & 0177 \\
\hline 17 & 1SH & $>5$ & 30 & 12 & $0.1 / 7$ \\
\hline 18 & HBA1C & $<=7$ & 57 & 3 & $*_{<} 00001$ \\
\hline 10 & Admission & $>7$ & 21 & 19 & $<0.0001$ \\
\hline
\end{tabular}

\begin{tabular}{|c|c|c|c|c|}
\hline \multicolumn{5}{|c|}{$\begin{array}{l}\text { Continuous Variable (Univariate analysis) for functional outcome } \\
\text { predictors at } 3 \text { months }\end{array}$} \\
\hline $\mathrm{SN}$ & Variable & $\begin{array}{c}\text { MRS } \\
0-2\end{array}$ & $\begin{array}{c}\text { MRS } \\
3-6\end{array}$ & P-Value \\
\hline 1 & Age & $\begin{array}{l}54.65 \pm \\
12.361\end{array}$ & $\begin{array}{l}61.23 \pm \\
11.505\end{array}$ & ${ }^{*} 0.028$ \\
\hline
\end{tabular}

of stroke severity and functional outcome of acute ischemic stroke in patients with diabetes mellitus.
Table 3: Logistic Regression model for predictors of stroke severity (NIHSS) at admission.

Logistic Regression model for predictors of stroke severity (NIHSS) at admission

\begin{tabular}{|l|c|c|c|c|c|c|}
\hline \multirow{2}{*}{ Parameter } & Parameter & Standard & $\begin{array}{c}\text { Odds } \\
\text { Ratio }\end{array}$ & \multicolumn{2}{|c|}{$\begin{array}{c}\text { 95\% Confidence } \\
\text { Intervals }\end{array}$} & P-Value* \\
\cline { 2 - 7 } & Estimate & $\begin{array}{c}\text { Error } \\
\text { (SE) }\end{array}$ & $\mathbf{( O R )}$ & & \\
\cline { 2 - 7 } & $\mathbf{( \beta )}$ & & & Lower & Upper & \\
\hline Alcohol & -3.667 & 1.131 & 0.026 & 0.003 & 0.235 & 0.001 \\
\hline HBA1C & -4.799 & 1.108 & 0.008 & 0.001 & 0.072 & $<0.0001$ \\
Admission & & & & & & \\
\hline
\end{tabular}

HDL: High density lipoprotein; LDL: Low density lipoprotein; VLDL: Very low density lipoprotein; TGL: Triglycerides; FPG: Fasting plasma glucose; PPG: Post prandial plasma glucose; TSH: Thyroid stimulating hormone; NIHSS: National institute of health stroke score; mRS: Modified rank in score; HbA1c: Glycated hemoglobin; ADM: Admission

The current study reports a prospective study of 100 patients recruited from emergency \& outpatient departments of Neurology who were admitted with acute ischemic stroke and diabetes. As in other studies [4], the current study also showed male predominance. The accumulation of traditional risk factors and along with aging in males is likely to explain the male predominance among acute ischemic stroke patients.

The current study has shown smoking had significant statistical correlation both with the severity at admission and functional outcome at 3 months among the diabetes patients. Besides smokers have a propensity for poor glycemic control, similar finding was noted by other studies [5-7]. There is much evidence that smoking increases the risk of diabetes. Smoking is associated with central obesity. Smoking also increases inflammation and oxidative stress, to directly damage $\beta$-cell function and to impair endothelial function.

Alcohol had also been associated with increased stroke severity and poor functional outcome in diabetes with acute ischemic stroke. Alcoholics were having poor glycemic control which is consistent with other series reported by some authors [8-10]. Diabetes mellitus is recognized clinically as a complication of alcoholism. Heavy amounts of alcohol show direct diabetogenic effects with its contribution to excess caloric intake and obesity, induction of pancreatitis, disturbance of the carbohydrate and glucose metabolism and the impairment of the liver function, which affects the blood glucose levels.

TSH was higher among diabetic patients yet it was not found to have influence over the severity and functional outcome of acute ischemic stroke.

The exact mechanism by which poor glycemic control with (elevated $\mathrm{HbA1c}$ and increased FPG and PPG) affects severity and outcome of ischemic stroke patients is less clear. General complications related to poorly controlled glycemic 
status could be one explanation. An increased HbA1C level reflects poor long term glycemic control and has its specific implications on the structure and function of vascular bed including small as large cerebral vessels. Increased $\mathrm{HbA1c}$ level might also be a marker of poor compliance indicating an unhealthy life style.

Evidence is compelling that increased stroke risk is associated with high levels of total cholesterol and low-density lipoprotein, and decreased high-density lipoprotein levels. In the Helsinki young stroke registry [11], dyslipidemia was clearly the most prominent well documented risk factor. The present study also found high mean levels of total cholesterol (P-0.017) and VLDL (P-0.03), and TGL (P-0.001) levels in patients of ischemic stroke, especially with poor glycemic control.

In our study we were not able to find significance of TOAST etiological classification to predict the severity (NIHSS) and functional outcome (mRS) among the diabetic ischemic stroke patients.

Kizer et al. [12] studied the relationship between HbA1c and stroke. The results had shown that after adjusting age, gender, smoking, blood lipids and other variances, $\mathrm{HbA} 1 \mathrm{c}$ and stroke risk was significantly associated [12-15]. They emphasized that strict control of glycated haemoglobin might be benefit for stroke prevention in patients with diabetes. The present study had shown that FPG, PPG values and HbA1c on admission had affected the NIHSS score, three months mRS score significantly. Patients with high $\mathrm{HbAlc}$, high FPG, high PPG had high NIHSS score at admission with poor outcomes at 3 months $(\mathrm{P}<0.001)$. That is to say, a higher HbA1c levels has a more serious neurological impairment, and the condition might be more serious. So, $\mathrm{HbA1c}$ level at admission might be an important predictor to evaluate the neurological impairment in patients with acute ischemic stroke. And a higher HbA1c level has a more serious neurological impairment on admission and the prognosis is worse after three months. The mechanism might be associated with long-term high blood glucose and high blood HbA1c, which lead to lesions of large blood vessels and which lead to oxygen dissociation curve to the left, resulting in oxygen dissociation barrier, nerve tissue ischemia and hypoxia, that is not benefit for the recovery of neurological function, and the prognosis is worse. This result is in line with the result of Kamouchi et al. [16], who studied 3627 patients, the result showed that neurological improvement is lower relevant to age and sex and is higher relevant to the blood HbA1c level on admission. In summary, the current study suggests that blood $\mathrm{HbA1c}$ levels on admission may influence severity in patients with acute ischemic stroke and may adversely predict three months prognosis. So, HbA1c level maybe is an important predictor to evaluate the neurological impairment and three months prognosis in patients of acute ischemic stroke with diabetes.

Therefore, effectively lowering blood HbA1c levels may reduce the severity of neurological impairment in patients with acute ischemic stroke, and may be can improve the quality of life in patients with acute ischemic stroke.
When we analyzed the risk factors effect on the severity of stroke in isolation, we observed only the alcohol and the HbA1c at admission were found to have the statistically significant correlation to the severity of stroke. We were not able to find any statistical association of individual risk factors with the stroke functional outcome at the follow up period. It suggests that the clustering of the risk factors have more impact on the functional outcome rather than that of each factor in isolation.

Limitation of this study is that we did not measure the size of the stroke lesion by CT or MRI, however it is well known that NIHSS score is a good clinical severity measure, which parallels infarct volume. We have not considered diabetic complications and complications related to other systems during follow up period which will affect the prognosis.

\section{Conclusion}

Based on results from this prospective study, clustering of factors like age, smoking, alcohol, TGL, FPG, PPG and $\mathrm{HbA1c}$ were found to have significant influence, for the severity of stroke at admission and functional outcome in diabetic patients with acute ischemic stroke. Whereas alcohol and $\mathrm{HbA} 1 \mathrm{c}$ at admission were found to have independent effect on the stroke severity. But none of the risk factors studied were having independent impact on the stroke functional outcome at the follow up. Further studies are needed to elucidate whether treatment to address these risk factors before onset of stroke improves clinical course and outcome of acute ischemic stroke in patients with diabetes.

\section{References}

1. Goyal A, Yusuf S. 2007. The burden of cardiovascular disease in the Indian subcontinent. Indian J Med Res 124(3): 235-244.

2. Libby P. 2005. Prevention and treatment of atherosclerosis. In: Kasper DL, Braunwald E, Fauci AS, Hauser SL, Longo DL, et al. (eds) Harrison's Principles of Internal Medicine, $16^{\text {th }}$ edition. McGraw-Hill Education, USA, pp 1430-1433.

3. Murray CJ, Lopez AD. 1997. Global mortality, disability, and the contribution of risk factors: Global Burden of Disease Study. Lancet 349(9063): 1436-1442. doi: 10.1016/S0140-6736(96)07495-8

4. Smith MA, Lisabeth LD, Brown DL, Morgenstern LB. 2005. Gender comparisons of diagnostic evaluation for ischemic stroke patients. Neurology 65(6): 855-858. doi: 10.1212/01.wnl.0000176054.72325.0f

5. Chang SA. 2012. Smoking and type 2 diabetes mellitus. Diabetes Metab J36(6): 399-403. doi: 10.4093/dmj.2012.36.6.399

6. Prasad S, Cucullo L. 2015. Impact of tobacco smoking and type-2 diabetes mellitus on public health: a cerebrovascular perspective. $J$ Pharmacovigil Suppl 2: e003. doi: 10.4172/2329-6887.S2-e003

7. Eliasson B. 2003. Cigarette smoking and diabetes. Prog Cardiovasc Dis 45(5): 405-413. doi: 10.1053/pcad.2003.00103

8. van de Wiel A. 2004. Diabetes mellitus and alcohol. Diabetes Metab Res Rev 20(4): 263-267. doi: 10.1002/dmrr.492

9. Kim SJ, Kim DJ. 2012. Alcoholism and diabetes mellitus. Diabetes Metab J 36(2): 108-115. doi: 10.4093/dmj.2012.36.2.108

10. Wannamethee SG, Shaper AG, Perry IJ, Alberti KG. 2002. Alcohol consumption and the incidence of type II diabetes. $J$ Epidemiol Community Health 56(7): 542-548. doi: 10.1136/jech.56.7.542

11. Putaala J, Metso AJ, Metso TM, Konkola N, Kraemer Y, et al. 2009. Analysis of 1008 consecutive patients aged 15 to 49 with first-ever 
ischemic stroke: the Helsinki young stroke registry. Stroke 40(4): 11951203. doi: 10.1161/STROKEAHA.108.529883

12. Kizer JR, Wiebers DO, Whisnant JP. 2006. Glycernic level and future stroke in type 2 diabetes mellitus: the strong heart study. Circulation 114(18): 444-446.

13. Tuomilehto J, Rastenyte D, Jousilahti P, Sarti C, Vartiainen E. 1996. Diabetes mellitus as a risk factor for death from stroke. Prospective study of the middle-aged Finnish population. Stroke 27(2): 210-215. doi: 10.1161/01.STR.27.2.210

14. Megherbi SE, Milan C, Minier D, Couvreur G, Osseby GV, et al.
2003. Association between diabetes and stroke subtype on survival and functional outcome 3 months after stroke: data from the European BIOMED stroke project. Stroke 34(3): 688-694. doi: 10.1161/01. STR.0000057975.15221.40

15. Mankovsky BN, Ziegler D. 2004. Stroke in patients with diabetes mellitus. Diabetes Metab Res Rev 20(4):268-287. doi: 10.1002/dmrr.490

16. Kamouchi M, Matsuki T, Hata J, Kuwashiro T, Ago T, et al. 2011. Prestroke glycemic control is associated with the functional outcome in acute ischemic stroke: the Fukuoka stroke registry. Stroke 42(10): 27882794. doi: 10.1161/STROKEAHA.111.617415 